\title{
REGULARITY OF CUBIC GRAPH WITH APPLICATION
}

\author{
Kishore Kumar.P.K ${ }^{1}$, Hossein Rashmanlou ${ }^{2}$, A.A. Talebi ${ }^{3}$, F. Mofidnakhaei ${ }^{4}$ \\ ${ }^{1}$ Department of Mathematics, Al Musanna College of Technology, \\ Sultanate of Oman \\ kishorePK@act.edu.om \\ ${ }^{2}$ Department of Computer Science, University College of Adib, \\ Sari, Iran \\ rashmanlou.1987@gmail.com \\ ${ }^{3}$ Department of Mathematics, University of Mazandaran, Babolsar, Iran \\ a.talebi@umz.ac.ir \\ ${ }^{4}$ Department of Physics, Sari Branch, Islamic Azad University, Sari, Iran \\ Farshid.Mofidnakhaei@gmail.com
}

\begin{abstract}
A cubic graph is a generalized structure of a fuzzy graph that gives more precision, flexibility and compatibility to a system when compared with systems that are designed using fuzzy graphs. In this paper, some properties of an edge regular cubic graph are given. Particularly, strongly regular, edge regular and bi-regular cubic graphs are defined and the necessary and sufficient condition for a cubic graph to be strongly regular is studied. Likewise, we have introduced a partially edge regular cubic graph and fully edge regular cubic graph with suitable illustrations. Finally, we gave an application of cubic digraph in travel time.
\end{abstract}

Key words and Phrases: Cubic graph, strongly regular cubic graph, bi-regular cubic graph

Abstrak. Graf kubik merupakan suatu struktur perumuman dari graf fuzzy yang memberikan banyak presisi, fleksibilitas, dan kompatibilitas terhadap suatu sistem yang dirancang menggunakan graf fuzzy. Dalam paper ini, dipelajari beberapa sifat dari suatu graf kubik yang teratur sisi. Didefinisikan pula graf kubik teratur kuat yang teratur sisi dan bi-regular serta dipelajari syarat perlu dan syarat cukup bagi suatu graf kubik agar menjadi graf yang teratur kuat. Kemudian, diperkenalkan graf kubik yang teratur sisi secara parsial dan graf kubik teratur sisi secara penuh dengan beberapa ilustrasi diberikan. Terakhir, diberikan aplikasi dari graf kubik pada travel time.

Kata kunci: Graf kubik, Graf kubik teratur kuat, Graf kubik bi-regular.

2000 Mathematics Subject Classification: 05C72, 05 C99. Received: 25-08-2017, revised : 20-05-2018, accepted: 31-05-2018. 


\section{INTRODUCTION}

Zadeh introduced the concept of fuzzy set in his seminal paper [22] of 1965. A fuzzy set of a universe $\mathrm{X}$ is a function from $\mathrm{X}$ into the unit closed interval $[0,1]$ of real number. In [23] Zadeh made an extension of the concept of a fuzzy set by an interval-valued fuzzy set, i.e., a fuzzy set with an interval-valued membership function. The first definition of fuzzy graphs was proposed by Kaufmann [5] in 1973. Interval-valued fuzzy sets have been actively used in real-life applications. For example, Sambuc [14] in medical diagnosis in thyroidian pathology, Kohout [7] also in medicine, Turksen in preferences modeling [21], etc. These works and others showed the importance of these sets. Jun et al. [4] introduced cubic sets. The fuzzy graph theory as a generalization of Euler's graph theory was first introduced by Rosenfeld [13] in 1975. Later, Bhattacharya [2] gave some remarks on fuzzy graphs and some operations on fuzzy graphs were introduced by Mordeson and Peng [8]. The complement of a fuzzy graph was defined by Mordeson [9] and further studied by Sunitha and Vijayakumar [15]. Hongmei and Lianhua gave the definition of interval-valued fuzzy graphs [3]. Akram and Dudek defined some operations on intervalvalued fuzzy graphs [1]. Rashmanlou et al. [10, 11, 12] introduced some properties of highly irregular interval-valued fuzzy graphs, and new concepts of bipolar fuzzy graphs. Karunambigai et al. [6] introduced edge regular intuitionistic fuzzy graph. Samanta and Pal $[15,16,17,18,19,20]$ defined fuzzy tolerance graph, fuzzy threshold graph, fuzzy kcompetition graph and p-competition fuzzy graph and new concepts of fuzzy planar graph. The major role of cubic graph theory in computer applications is the development of graph algorithms. These algorithms are used to solve problems that are modeled in the form of graphs and the corresponding computer science application problems. One of the most widely studied classes of cubic graphs is regular cubic graphs. Theoretical concepts of cubic graphs are highly utilized by computer science applications. Especially in research areas of computer science such as data mining, image segmentation, clustering, image capturing and networking. The cubic graphs are more flexible and compatible than fuzzy graphs due to the fact that they have many applications in networks. They show up in many contexts. Fore example, r-regular cubic graphs with connectivity and edge-connectivity equal to $r$ play a key role in designing reliable communication networks. Hence, in this paper some properties of an edge regular cubic graph are given. Particularly, strongly regular, edge regular and biregular cubic graphs are defined and the necessary and sufficient condition for a cubic graph to be strongly regular is studied. Also, we have introduced a partially edge regular cubic graph and fully edge regular cubic graph with suitable illustrations. After introductory Section 1, some basic definitions are given in Section 2. In Section 3, the concepts of regularity of cubic graphs are defined. In Section 4, an application is given in travel time. At last conclusion is given in Section 5.

\section{PRELIMINARIES}

A graph is an ordered pair $G=(V, E)$, where $V$ is the set of vertices of $G$ and $E$ is the set of edges of $G$. A subgraph of a graph $G=(V, E)$ is a graph $H=(W, F)$, where $W \subseteq V$ and $F \subseteq E$. A fuzzy graph $G=(\sigma, \mu)$ is a pair of functions $\sigma: V \rightarrow[0,1]$ and $\mu: V \times V \rightarrow[0,1]$ with $\mu(u, v) \leq \sigma(u) \wedge \sigma(v)$, for all $u, v \in V$, where $V$ is a finite non-empty set and $\wedge$ denote minimum. We introduce below necessary notions and present 
a few auxiliary results that will be used throughout the paper.

A map $\lambda: X \rightarrow[0,1]$ is called a fuzzy subset of $X$. For any two fuzzy subsets $\lambda$ and $\mu$ of $X, \lambda \subseteq \mu$ means that, for all $x \in X, \lambda(x) \leq \mu(x)$. The symbol $\lambda \wedge \mu$ and $\lambda \vee \mu$ will mean the following fuzzy subsets of $X$.

$(\lambda \wedge \mu)(x)=\lambda(x) \wedge \mu(x)$ and $(\lambda \vee \mu)(x)=\lambda(x) \vee \mu(x)$, for all $x \in X$.

Let $X$ be a non-empty set. A function $A: X \rightarrow[I]$ is called an interval-valued fuzzy set (shortly, an IVF set) in $X$. Let $[I]^{X}$ stands for the set of all IVF sets in $X$. For every $A \in[I]^{X}$ and $x \in X, A(x)=\left[A^{-}(x), A^{+}(x)\right]$ is called the degree of membership of an element $x$ to $A$, where $A^{-}: X \rightarrow I$ and $A^{+}: X \rightarrow I$ are fuzzy sets in $X$ which are called a lower fuzzy set and an upper fuzzy set in $X$, respectively. For simplicity, we denote $A=\left[A^{-}, A^{+}\right]$. For every $A, B \in[I]^{X}$, we define $A \subseteq B$ if and only if $A(x) \leq B(x)$, for all $x \in X$.

Definition 2.1. Let $A=\left[A^{-}, A^{+}\right]$, and $B=\left[B^{-}, B^{+}\right]$be two interval-valued fuzzy set in $X$. Then we define $\operatorname{rmin}\{A(x), B(x)\}=$ $\left[\min \left\{A^{-}(x), B^{-}(x)\right\}, \min \left\{A^{+}(x), B^{+}(x)\right\}\right]$, $\operatorname{rmax}\{A(x), B(x)\}=\left[\max \left\{A^{-}(x), B^{-}(x)\right\}\right.$, $\left.\max \left\{A^{+}(x), B^{+}(x)\right\}\right]$.

Definition 2.2. Let $X$ be a non-empty set. By a cubic set in $X$, we mean a structure $A=\{<$ $x, A(x), \lambda(x): x \in X>\}$ in which $A$ is an interval-valued fuzzy sets in $X$ and $\lambda$ is a fuzzy set in $X$. A cubic set $A=\{\langle x, A(x), \lambda(x): x \in X\rangle\}$ is simply denoted by $A=\langle A, \lambda\rangle$. The collection of all cubic sets in $X$ is denoted by $C P(X)$.

Definition 2.3. A cubic graph is a triple $G=\left(G^{*}, P, Q\right)$ where $G^{*}=(V, E)$ is a graph, $P=\left(\widetilde{\mu_{P}}, \lambda_{P}\right)$ is a cubic set on $V$ and $Q=\left(\widetilde{\mu_{Q}}, \widetilde{\mu_{Q}}\right)$ is a cubic set on $V \times V$ such that $\widetilde{\mu_{Q}}(x y) \leq \operatorname{rmin}\left\{\widetilde{\mu_{P}}(x), \widetilde{\mu_{P}}(y)\right\}$ and $\widetilde{\lambda_{Q}}(x y) \geq \operatorname{rmax}\left\{\widetilde{\lambda_{P}}(x)\right.$, $\left.\widetilde{\lambda_{P}}(y)\right\}$

The underlying crisp graph of a cubic graph $G=(A, B)$, is the graph $G=(V, E)$, where $V=\left\{v: \widetilde{\mu_{P}}>0\right.$ and $\left.\lambda_{P}>0\right\}$ and $E=\left\{\{u, v\}: \widetilde{\mu_{Q}}(\{u, v\})>0, \widetilde{\mu_{Q}}(\{u, v\})>0\right\}$. $V$ is called the vertex set and $E$ is called the edge set. A cubic graph maybe also denoted as $G=(V, E)$.

Definition 2.4. A cubic graph $G=\left(G^{*}, P, Q\right)$ is called complete if $\widetilde{\mu_{Q}}(x y)=r \operatorname{rin}\left\{\mu_{P}(x), \mu_{P}(y)\right\}$ and $\widetilde{\lambda_{Q}}(x y)=\operatorname{rmax}\left\{\widetilde{\lambda_{P}}(x)\right.$,

$\left.\widetilde{\lambda_{P}}(y)\right\}$ for all $x, y \in V$.

Definition 2.5. A cubic graph $G=\left(G^{*}, P, Q\right)$ is called strong if $\widetilde{\mu_{Q}}(x y)=\operatorname{rmin}\left\{\mu_{P}(x), \mu_{P}(y)\right\}$ and $\widetilde{\lambda_{Q}}(x y)=\operatorname{rmax}\left\{\widetilde{\lambda_{P}}(x), \widetilde{\lambda_{P}}(y)\right\}$ for all $x y \in E$.

Definition 2.6. The complement of a cubic graph $G=(A, B)$ is a cubic graph $\bar{G}=$ $\left(\overline{G^{*}}, \bar{P}, \bar{Q}\right)$, where $\bar{P}=\left(\overline{\widetilde{\mu_{P}}}, \overline{\lambda_{P}}\right)$ and $\bar{Q}=\left(\overline{\widetilde{\mu_{Q}}}, \overline{\lambda_{Q}}\right)$ is defined by: $\overline{\widetilde{\mu_{Q}}}(x y)=\operatorname{rmin}\left\{\mu_{P}(x), \mu_{P}(y)\right\}-$ $\widetilde{\mu_{Q}}(x y)$ and $\overline{\widetilde{\lambda_{Q}}}(x y)=\widetilde{\lambda_{Q}}(x y)-\operatorname{rmax}\left\{\widetilde{\lambda_{P}}(x), \widetilde{\lambda_{P}}(y)\right\}$

Definition 2.7. Let $G=(V, E)$ be a cubic graph.

(i) The neighborhood degree of a vertex $v$ is defined as $D_{N}(v)=\left(d_{N_{\widetilde{\mu p}}}(v), d_{N_{\lambda_{Q}}}(v)\right)$, where $d_{N_{\mu_{P}}}(v)=\left(\sum_{w \in N\left(\widetilde{\mu_{p}^{-}}\right)} \widetilde{\mu_{P}^{-}}(w), \sum_{w \in N\left(\mu_{P}^{+}\right)} \mu_{P}^{+}(w)\right)$ and $d_{N_{\lambda_{Q}}}(v)=\sum_{w \in N\left(\lambda_{Q}\right)} \lambda_{Q}(w)$.

(ii) The degree of a vertex $v_{i}$ is defined by $d_{G}\left(v_{i}\right)=\left(d_{\widetilde{\mu_{P}}}\left(v_{i}\right), d_{\lambda_{Q}}\left(v_{i}\right)\right)=\left(k_{1}, k_{2}\right)$, where 


$$
\begin{aligned}
& k_{1}=d_{\widetilde{\mu_{P}}}\left(v_{i}\right)=\left(\sum_{v_{i} \neq v_{j}} \widetilde{\mu_{Q}^{-}}\left(v_{i} v_{j}\right), \sum_{v_{i} \neq v_{j}} \widetilde{\mu_{Q}^{+}}\left(v_{i} v_{j}\right)\right) \\
& \text { and } k_{2}=d_{\lambda_{Q}}\left(v_{i}\right)=\sum_{v_{i} \neq v_{j}} \lambda_{Q}\left(v_{i} v_{j}\right) .
\end{aligned}
$$

Definition 2.8. A cubic graph $G=(V, E)$ is said to be

(i) $\left(k_{1}, k_{2}\right)$-regular if $d_{G}\left(v_{i}\right)=\left(k_{1}, k_{2}\right)$, for all $v_{i} \in V$ and also $G$ is said to be a regular cubic graph of degree $\left(k_{1}, k_{2}\right)$.

(ii) bipartite if the vertex set $V$ can be partitioned into two non-empty sets $V_{1}$ and $V_{2}$ such that

(a) $\widetilde{\mu_{Q}}\left(v_{i} v_{j}\right)=0$ and $\lambda_{Q}\left(v_{i} v_{j}\right)=0$, if $\left(v_{i}, v_{j}\right) \in V_{1}$ or $\left(v_{i}, v_{j}\right) \in V_{2}$

(b) $\widetilde{\mu_{Q}}\left(v_{i} v_{j}\right)=0, \lambda_{Q}\left(v_{i} v_{j}\right)>0$, if $v_{i} \in V_{1}$ or $v_{j} \in V_{2}$

(c) $\widetilde{\mu_{Q}}\left(v_{i} v_{j}\right)>0, \lambda_{Q}\left(v_{i} v_{j}\right)=0$, if $v_{i} \in V_{1}$ or $v_{j} \in V_{2}$, for some $i$ and $j$.

Definition 2.9. Let $G^{*}=(V, E)$ be a crisp graph and let $e=v_{i} v_{j}$ be an edge in $G^{*}$. Then, the degree of an edge $e=v_{i} v_{j} \in E$ is defined as $d_{G^{*}}\left(v_{i} v_{j}\right)=d_{G^{*}}\left(v_{i}\right)+d_{G^{*}}\left(v_{j}\right)-2$.

Definition 2.10. (i) The order of $G$ is defined to be $O(G)=\left(O_{\widetilde{\mu_{P}}}, O_{\lambda_{P}}\right)$, where $O_{\widetilde{\mu_{P}}}=$ $\sum_{u \in V} \overparen{\mu_{P}(u)}$ and $O_{\lambda_{P}}=\sum_{u \in V} \lambda_{P}(u)$.

(ii) The size of $G$ is defined to be

$S(G)=\left(S_{\widetilde{\mu_{Q}}}(G), S_{\lambda_{Q}}(G)\right)$, where $S_{\widetilde{\mu_{Q}}}(G)=\sum_{u \neq v} \widetilde{\mu_{Q}(u v)}$ and $S_{\lambda_{Q}}(G)=\sum_{u \neq v} \lambda_{Q}(u v)$.

3. ISOMORPHIC PROPERTIES OF NEIGHBORLY IRREGULAR AND HIGHLY IRREGULAR CUBIC GRAPHS

In this section, we define weak isomorphism, co-weak isomorphism and isomorphism of neighborly irregular cubic graphs and prove that.

Definition 3.1. Let $G=(V, E)$ be a cubic graph.

(i) The degree of an edge $e_{i j} \in E$ is defined as

$$
\begin{aligned}
& d_{\widetilde{\mu_{Q}}}\left(e_{i j}\right)=d_{\widetilde{\mu_{P}}}\left(v_{i}\right)+d_{\widetilde{\mu_{P}}}\left(v_{j}\right)-2 \widetilde{\mu_{Q}}\left(v_{i} v_{j}\right) \quad \text { or } d_{\widetilde{\mu_{Q}}}\left(e_{i j}\right)=\sum_{\substack{v_{i} v_{k} \in E \\
k \neq j}} \widetilde{\mu_{Q}}\left(v_{i} v_{k}\right)+\sum_{\substack{v_{k} v_{j} \in E \\
k \neq i}} \widetilde{\mu_{Q}}\left(v_{k} v_{j}\right) . \\
& d_{\lambda_{Q}}\left(e_{i j}\right)=d_{\lambda_{P}}\left(v_{i}\right)+d_{\lambda_{P}}\left(v_{j}\right)-2 \lambda_{Q}\left(v_{i} v_{j}\right) \text { or } d_{\lambda_{Q}}\left(e_{i j}\right)=\sum_{\substack{v_{i} v_{k} \in E \\
k \neq j}} \lambda_{Q}\left(v_{i} v_{k}\right)+\sum_{\substack{v_{k} v_{j} \in E \\
k \neq i}} \lambda_{Q}\left(v_{k} v_{j}\right) .
\end{aligned}
$$

(ii) The minimum edge degree of $G$ is $\delta_{E}(G)=\left(\delta_{\widetilde{\mu_{Q}}}(G), \delta_{\lambda_{Q}}(G)\right)$, where $\delta_{\widetilde{\mu_{Q}}}(G)=\wedge\left\{d_{\widetilde{\mu_{Q}}}\left(e_{i j}\right) \mid\right.$ $\left.e_{i j} \in E\right\}$ and $\delta_{\lambda_{Q}}(G)=\wedge\left\{d_{\lambda_{Q}}\left(e_{i j}\right) \mid e_{i j} \in E\right\}$.

(iii) The maximum edge degree of $G$ is $\Delta_{E}(G)=\left(\Delta_{\widetilde{\mu_{Q}}}(G), \Delta_{\lambda_{Q}}(G)\right)$, where $\Delta_{\widetilde{\mu_{P}}}(G)=$ $\vee\left\{d_{\widetilde{\mu_{Q}}}\left(e_{i j}\right) \mid e_{i j} \in E\right\}$ and $\Delta_{\lambda_{Q}}(G)=\vee\left\{d_{\lambda_{Q}}\left(e_{i j}\right) \mid e_{i j} \in E\right\}$.

(iv) The total edge degree of an edge $e_{i j} \in E$ is defined as

$t d_{\widetilde{\mu_{Q}}}\left(e_{i j}\right)=\sum_{\substack{v_{i}, v_{k} \in E \\ k \neq j}} \widetilde{\mu_{Q}}\left(v_{i} v_{k}\right)+\sum_{\substack{v_{k} v_{j} \in E \\ k \neq i}} \widetilde{\mu_{Q}}\left(v_{k} v_{j}\right)+\widetilde{\mu_{Q}}\left(e_{i j}\right), t d_{\lambda_{Q}}\left(e_{i j}\right)=\sum_{\substack{v_{i}, v_{k} \in E \\ k \neq j}} \lambda_{Q}\left(v_{i} v_{k}\right)+\sum_{\substack{v_{k}, v_{j} \in E \\ k \neq i}} \lambda_{Q}\left(v_{k} v_{j}\right)+$ $\lambda_{Q}\left(e_{i j}\right)$

(v) The edge degree of $G$ is defined by $d_{G}\left(e_{i j}\right)=\left(d_{\widetilde{\mu_{Q}}}\left(e_{i j}\right), d_{\lambda_{Q}}\left(e_{i j}\right)\right)$ and the total edge degree of $G$ is defined by $t d_{G}\left(e_{i j}\right)=\left(t d_{\widetilde{\mu_{Q}}}\left(e_{i j}\right), t d_{\lambda_{Q}}\left(e_{i j}\right)\right)$.

Example 3.1. Consider the cubic graph $G=(V, E)$ in Figure 3.1, where $V=\{a, b, c, d\}$ and $E=\{a b, a d, b c, b d, c d\}$. Then 


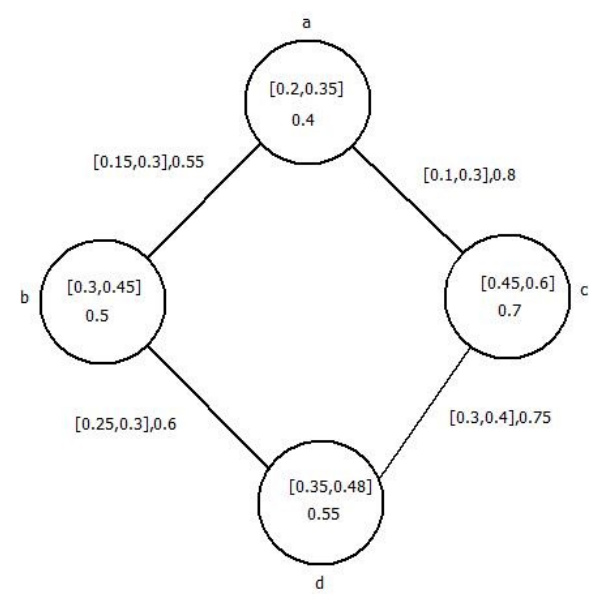

FIGURE 1. Cubic graph

$$
d_{\widetilde{\mu_{Q}}}(a c)=[0.7,1.05], d_{\lambda_{Q}}(a c)=1.1, d_{G}(u w)=[0.7,1.05], 1.1, t d_{\widetilde{\mu_{Q}}}(a c)=[0.7+
$$
$0.1=0.8,1.05+0.25=1.30]$ and $t d_{\lambda_{Q}}(a c)=1.7+0.4=2.1$. Hence, $t d_{G}(a c)=$ $[0.8,1.30], 2.1\left(e_{i j}=\left(u_{i}, u_{j}\right)\right)$.

Definition 3.2. Let $G=(V, E)$ be a cubic graph.

(i) If each edge in $G$ has the same degree $\left(l_{1}, l_{2}\right)$, then $G$ is said to be an edge regular cubic graph.

(ii) If each edge in $G$ has the same total degree $\left(t_{1}, t_{2}\right)$, then $G$ is said to be a totally edge regular cubic graph.

Example 3.2. Consider the cubic graph $G=(V, E)$ as in Figure 2, where $V=\left\{u_{1}, u_{2}, u_{3}, u_{4}\right\}$ and $E=\left\{u_{1} u_{2}, u_{1} u_{3}, u_{3} u_{4}, u_{2} u_{4}\right\}$. Then

$\bar{d}_{G}\left(e_{12}\right)=d_{G}\left(e_{24}\right)=d_{G}\left(e_{34}\right)=d_{G}\left(e_{13}\right)=[0.1,0.3], 1.1$.

Theorem 3.1. Let $G=(V, E)$ be a cubic graph on a cycle $G^{*}$. Then

$$
\sum_{v_{i} \in V} d_{G}\left(v_{i}\right)=\sum_{v_{i} v_{j} \in E} d_{G}\left(v_{i} v_{j}\right)
$$

Proof. Let $G=(V, E)$ be a cubic graph and $G^{*}$ be a cycle $v_{1} v_{2} v_{3} \cdots v_{n} v_{1}$. Then $\sum_{i=1}^{n} d_{G}\left(v_{i} v_{i+1}\right)=\left(\sum_{i=1}^{n} d_{\widetilde{\mu_{Q}}}\left(v_{i} v_{i+1}\right), \sum_{i=1}^{n} d_{\lambda_{Q}}\left(v_{i} v_{i+1}\right)\right)$. Also $\widetilde{\mu_{P}}=\left\{\mu_{P}^{-}, \mu_{P}^{+}\right\}$and $\widetilde{\mu_{Q}}=\left\{\mu_{Q}^{-}, \mu_{Q}^{+}\right\}$ Now we have

$$
\begin{aligned}
& \quad \sum_{i=1}^{n} d_{\widetilde{\mu_{Q}}}\left(v_{i} v_{i+1}\right)= \\
& d_{\widetilde{\mu_{Q}}}\left(v_{1} v_{2}\right)+d_{\widetilde{\mu_{Q}}}\left(v_{2} v_{3}\right)+\cdots+d_{\widetilde{\mu_{Q}}}\left(v_{n} v_{1}\right), \text { where } v_{n+1}=v_{1} \\
& =d_{\widetilde{\widetilde{P}}}\left(v_{1}\right)+d_{\widetilde{\mu_{P}}}\left(v_{2}\right)-2 \widetilde{\mu_{Q}}\left(v_{1} v_{2}\right)+d_{\widetilde{\mu_{P}}}\left(v_{2}\right)+d_{t}\left(v_{3}\right) \\
& =2 \widetilde{\mu_{P}}\left(v_{2} v_{3}\right)+\cdots+d_{\widetilde{\mu_{P}}}\left(v_{n}\right)+d_{\widetilde{\mu_{P}}}\left(v_{1}\right)-2 \widetilde{\mu_{Q}}\left(v_{n} v_{1}\right) \\
& =2 d_{\widetilde{\mu_{P}}}\left(v_{1}\right)+2 d_{\widetilde{\mu_{P}}}\left(v_{2}\right)+\cdots+2 d_{\widetilde{\mu_{P}}}\left(v_{n}\right)
\end{aligned}
$$




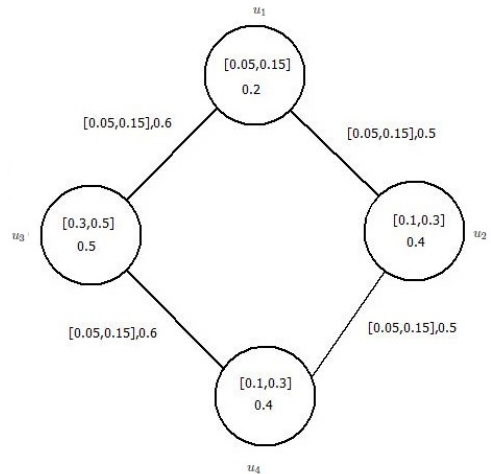

FiguRE 2. [0.1,0.3],1.1 - edge regular cubic graph

$=2\left(\widetilde{\mu_{Q}}\left(v_{1} v_{2}\right)+\widetilde{\mu_{Q}}\left(v_{2} v_{3}\right)+\cdots+\widetilde{\mu_{Q}}\left(v_{n} v_{1}\right)\right)$

$=2 \sum_{v_{i} \in V} d_{\widetilde{\mu_{p}}}\left(v_{i}\right)$

$-2 \sum_{i=1}^{n} \widetilde{\mu_{Q}}\left(v_{i} v_{i+1}\right)$

$=\sum_{v_{i} \in V} d_{\widetilde{\mu_{p}}}\left(v_{i}\right)+2 \sum_{i=1}^{n} \widetilde{\mu_{Q}}\left(v_{i} v_{i+1}\right)$

$-2 \sum_{i=1}^{n} \widetilde{\mu_{Q}}\left(v_{i} v_{i+1}\right)$

$=\sum_{v_{i} \in V} \widetilde{\mu_{P}}\left(v_{i}\right)$.

Similarly, $\sum_{i=1}^{n} d_{\lambda_{Q}}\left(v_{i} v_{i+1}\right)=\sum_{v_{i} \in V} d_{\lambda_{P}}\left(v_{i}\right)$.

Hence, $\sum_{i=1}^{n} d_{G}\left(v_{i} v_{i+1}\right)=\left(\sum_{v_{i} \in V} d_{\widetilde{\mu_{P}}}\left(v_{i}\right)\right.$,

$\left.\sum_{v_{i} \in V} d_{\lambda_{p}}\left(v_{i}\right)\right)$

$=\sum_{v_{i} \in V} d_{G}\left(v_{i}\right)$.

Remark 3.1. Let $G=(V, E)$ be a cubic graph on a crisp graph $G^{*}$. Then, $\sum_{v_{i} v_{j} \in E} d_{G}\left(v_{i} v_{j}\right)=$ $\left(\sum_{v_{i} v_{j} \in E} d_{G^{*}}\left(v_{i} v_{j}\right) \widetilde{\mu_{Q}}\left(v_{i} v_{j}\right)\right.$,

$\left.\sum_{v_{i} v_{j} \in E} d_{G^{*}}\left(v_{i} v_{j}\right) \lambda_{Q}\left(v_{i} v_{j}\right)\right)$, where $d_{G^{*}}\left(v_{i} v_{j}\right)=d_{G^{*}}\left(v_{i}\right)+d_{G^{*}}\left(v_{j}\right)-2$, for all $v_{i} v_{j} \in E$.

Theorem 3.2. Let $G=(V, E)$ be a cubic graph on a $k$-regular crisp graph $G^{*}$. Then, $\sum_{v_{i} v_{j} \in E} d_{G}\left(v_{i} v_{j}\right)=\left((k-1) \sum_{v_{i} \in V} d_{\widetilde{\mu p}}\left(v_{i}\right),(k-1) \sum_{v_{i} \in V} d_{\lambda_{P}}\left(v_{i}\right)\right)$.

Proof. By Remark 3.6, we have $\sum_{v_{i} v_{j} \in E} d_{G}\left(v_{i} v_{j}\right)$

$=\left(\sum_{v_{i} v_{j} \in E} d_{G^{*}}\left(v_{i} v_{j}\right) \widetilde{\mu_{Q}}\left(v_{i} v_{j}\right), \sum_{v_{i} v_{j} \in E}\right.$

$\left.d_{G^{*}}\left(v_{i} v_{j}\right) \lambda_{Q}\left(v_{i} v_{j}\right)\right)$

$=\left(\sum_{v_{i} v_{j} \in E}\left(d_{G^{*}}\left(v_{i}\right)+d_{G^{*}}\left(v_{j}\right)-2\right) \widetilde{\mu_{Q}}\left(v_{i} v_{j}\right)\right.$,

$\left.\sum_{v_{i} v_{j} \in E}\left(d_{G^{*}}\left(v_{i}\right)+d_{G^{*}}\left(v_{j}\right)-2\right) \lambda_{Q}\left(v_{i} v_{j}\right)\right)$. Since $G^{*}$ is a regular crisp graph, $d_{G^{*}}\left(v_{i}\right)=k$, for all $v_{i} \in V$ and so we have $\sum_{v_{i} v_{j} \in E} d_{G}\left(v_{i} v_{j}\right)=\left((k+k-2) \sum_{v_{i} v_{j} \in E} \widetilde{\mu_{Q}}\left(v_{i} v_{j}\right),(k+k-\right.$ 2) $\left.\sum_{v_{i} v_{j} \in E} \lambda_{Q}\left(v_{i} v_{j}\right)\right), \sum_{v_{i} v_{j} \in E} d_{G}\left(v_{i} v_{j}\right)=\left(2(k-1) \sum_{v_{i} v_{j} \in E} \widetilde{\mu_{Q}}\left(v_{i} v_{j}\right), 2(k-1) \sum_{v_{i} v_{j} \in E} \lambda_{Q}\left(v_{i} v_{j}\right)\right)$, $\sum_{v_{i} v_{j} \in E} d_{G}\left(v_{i} v_{j}\right)=\left((k-1) \sum_{v_{i} \in V} d_{\widetilde{\mu_{Q}}}\left(v_{i}\right),(k-1) \sum_{v_{i} \in V} d_{\lambda_{Q}}\left(v_{i}\right)\right)$. 
Theorem 3.3. Let $G=(V, E)$ be a cubic graph on a crisp graph $G^{*}$. Then, $\sum_{v_{i} v_{i} \in E} t d_{G}\left(v_{i} v_{j}\right)=$ $\left(\sum_{v_{i} v_{j} \in E} d_{G^{*}}\left(v_{i} v_{j}\right) \widetilde{\mu_{Q}}\left(v_{i} v_{j}\right)+\sum_{v_{i} v_{j} \in E} \widetilde{\mu_{Q}}\left(v_{i} v_{j}\right)\right.$, $\left.\sum_{v_{i} v_{j} \in E} d_{G^{*}}\left(v_{i} v_{j}\right) \lambda_{Q}\left(v_{i} v_{j}\right)+\sum_{v_{i} v_{j} \in E} \lambda_{Q}\left(v_{i} v_{j}\right)\right)$.

Proof. By definition of total edge degree of $G$, we have $\sum_{v_{i} v_{j} \in E} t d_{G}\left(v_{i} v_{j}\right)=$ $\left(\sum_{v_{i} v_{j} \in E} t d_{\widetilde{\mu_{Q}}}\left(v_{i} v_{j}\right), \sum_{v_{i} v_{j} \in E} t d_{\lambda_{Q}}\left(v_{i} v_{j}\right)\right)$ $=\left(\sum_{v_{i} v_{j} \in E}\left(d_{\widetilde{\mu_{Q}}}\left(v_{i} v_{j}\right)+\widetilde{\mu_{Q}}\left(v_{i} v_{j}\right)\right)\right.$, $\left.\sum_{v_{i} v_{j} \in E}\left(d_{\lambda_{Q}}\left(v_{i} v_{j}\right)+\lambda_{Q}\left(v_{i} v_{j}\right)\right)\right)$ $=\left(\sum_{v_{i} v_{j} \in E} d_{\widetilde{\mu_{Q}}}\left(v_{i} v_{j}\right)+\right.$ $\sum_{v_{i} v_{j} \in E} \widetilde{\mu_{Q}}\left(v_{i} v_{j}\right)$, $\left.\sum_{v_{i} v_{j} \in E} d_{\lambda_{Q}}\left(v_{i} v_{j}\right)+\sum_{v_{i} v_{j} \in E} \lambda_{Q}\left(v_{i} v_{j}\right)\right)$.

By Remark 3.6, we get

$$
\sum_{v_{i} v_{j} \in E} t d_{G}\left(v_{i} v_{j}\right)
$$

$=\left(\sum_{v_{i} v_{j} \in E} d_{G^{*}}\left(v_{i} v_{j}\right) \widetilde{\mu_{Q}}\left(v_{i} v_{j}\right)+\right.$

$\sum_{v_{i} v_{j} \in E} \widetilde{\mu_{Q}}\left(v_{i} v_{j}\right), \sum_{v_{i} v_{j} \in E} d_{G^{*}}\left(v_{i} v_{j}\right) \lambda_{Q}\left(v_{i} v_{j}\right)$

$\left.+\sum_{v_{i} v_{j} \in E} \lambda_{Q}\left(v_{i} v_{j}\right)\right)$.

Theorem 3.4. Let $G=(V, E)$ be a cubic graph. Then $\left(t_{B}, f_{B}\right)$ is a constant function if and only if the following are equivalent.

(i) $G$ is a edge regular cubic graph.

(ii) $G$ is totally edge regular cubic graph.

Proof. Assume that $\left(\widetilde{\mu_{P}}, \lambda_{Q}\right)$ is a constant function. Then $\widetilde{\mu_{Q}}\left(v_{i} v_{j}\right)=c_{1}$ and $\lambda_{Q}\left(v_{i} v_{j}\right)=$ $c_{2}$, for every $v_{i} v_{j} \in E$, where $c_{1}$ and $c_{2}$ are constants. Let $G$ be an $\left(l_{1}, l_{2}\right)$-edge regular cubic graph. Then, for all $v_{i} v_{j} \in E, d_{G}\left(v_{i} v_{j}\right)=\left(l_{1}, l_{2}\right)$ and $t d_{G}\left(v_{i} v_{j}\right)=\left(d_{\widetilde{\mu_{p}}}\left(v_{i} v_{j}\right)+\right.$ $\left.\widetilde{\mu_{Q}}\left(v_{i} v_{j}\right), d_{\lambda_{Q}}\left(v_{i} v_{j}\right)+\lambda_{Q}\left(v_{i} v_{j}\right)\right)=\left(l_{1}+c_{1}, l_{2}+c_{2}\right)$, for all $v_{i} v_{j} \in E$. Then $G$ is a totally edge regular. Now, let $G$ be a $\left(t_{1}, t_{2}\right)$-totally edge regular cubic graph. Then $\operatorname{td}_{G}\left(v_{i} v_{j}\right)=\left(t_{1}, t_{2}\right)$, for all $v_{i} v_{j} \in E$. So, we have $t d_{G}\left(v_{i} v_{j}\right)=\left(d_{\widetilde{\mu_{0}}}\left(v_{i} v_{j}\right)+\widetilde{\mu_{P}}\left(v_{i} v_{j}\right), d_{\lambda_{Q}}\left(v_{i} v_{j}\right)+\lambda_{Q}\left(v_{i} v_{j}\right)\right)=\left(t_{1}, t_{2}\right)$. Hence, $\left(d_{\widetilde{\mu_{Q}}}\left(v_{i} v_{j}\right), d_{\lambda_{Q}}\left(v_{i} v_{j}\right)\right)=\left(t_{1}-\widetilde{\mu_{Q}}\left(v_{i} v_{j}\right), t_{2}-\lambda_{Q}\left(v_{i} v_{j}\right)\right)=\left(t_{1}-c_{1}, t_{2}-c_{2}\right)$. Then, $G$ is a $\left(t_{1}-c_{1}, t_{2}-c_{2}\right)$ edge regular cubic graph.

Conversely, assume that $(i)$ and $(i i)$ are equivalent. We have to prove that $\left(\widetilde{\mu_{P}}, \lambda_{Q}\right)$ is a constant function. Suppose that $\left.\widetilde{\mu_{P}}, \lambda_{Q}\right)$ is not a constant function. Then $\widetilde{\mu_{Q}}\left(v_{i} v_{j}\right) \neq$ $\widetilde{\mu_{Q}}\left(v_{r} v_{s}\right)$ and $\lambda_{Q}\left(v_{i} v_{j}\right) \neq \lambda_{Q}\left(v_{r} v_{s}\right)$ for at least one pair of $v_{i} v_{j}, v_{r} v_{s} \in E$. Let $G$ be an $\left(l_{1}, l_{2}\right)$ edge regular cubic graph. Then, $d_{G}\left(v_{i} v_{j}\right)=d_{G}\left(v_{r} v_{s}\right)=\left(l_{1}, l_{2}\right)$. Hence for all $v_{i} v_{j} \in E$ and for all $v_{r} v_{s} \in E ; t d_{G}\left(v_{i} v_{j}\right)=\left(d_{\widetilde{\mu_{Q}}}\left(v_{i} v_{j}\right)+\widetilde{\mu_{Q}}\left(v_{i} v_{j}\right), d_{\lambda_{Q}}\left(v_{i} v_{j}\right)+\lambda_{Q}\left(v_{i} v_{j}\right)\right)=$ $\left(l_{1}+\widetilde{\mu_{Q}}\left(v_{i} v_{j}\right), l_{2}+\lambda_{Q}\left(v_{i} v_{j}\right)\right) t d_{G}\left(v_{r} v_{s}\right)=\left(\widetilde{\mu_{Q}}\left(v_{r} v_{s}\right)+\widetilde{\mu_{Q}}\left(v_{r} v_{s}\right), d_{\lambda_{Q}}\left(v_{r} v_{s}\right)+\lambda_{Q}\left(v_{r} v_{s}\right)\right)=\left(l_{1}\right.$ $\left.+\widetilde{\mu_{Q}}\left(v_{r} v_{s}\right), l_{2}+\lambda_{Q}\left(v_{r} v_{s}\right)\right)$ Since, $\widetilde{\mu_{Q}}\left(v_{i} v_{j}\right) \neq \widetilde{\mu_{Q}}\left(v_{r} v_{s}\right)$ and $\lambda_{Q}\left(v_{i} v_{j}\right) \neq \lambda_{Q}\left(v_{r} v_{s}\right)$, we have $t_{G}\left(v_{i} v_{j}\right) \neq t d_{G}\left(v_{r} v_{s}\right)$. Hence, $G$ is not a totally edge regular that is contradiction to our assumption. Therefore, $\left(\widetilde{\mu_{P}}, \lambda_{Q}\right)$ is a constant function. Similarly we can show that $\left(\widetilde{\mu_{P}}, \lambda_{Q}\right)$ is a constant function, when $G$ is a totally edge regular cubic graph.

Theorem 3.5. Let $G=(V, E)$ be a cubic graph on a $k$-regular crisp graph $G^{*}$. Then, $\left(t_{B}, f_{B}\right)$ is a constant if and only if $G$ is both regular and edge regular cubic graph. 


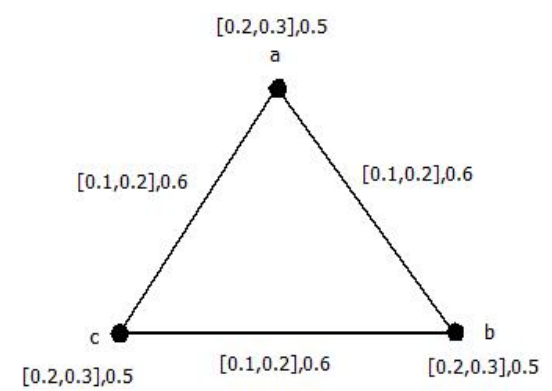

FIGURE 3. Strongly regular cubic graph

Proof. Let $G=(V, E)$ be a cubic graph on $G^{*}$ and let $G^{*}$ be a $k$-regular crisp graph. Assume that $\widetilde{\mu_{Q}}$ and $\lambda_{Q}$ are constant functions, i.e., $\widetilde{\mu_{Q}}\left(v_{i} v_{j}\right)=c$ and $\lambda_{Q}\left(v_{i} v_{j}\right)=t$, for all $v_{i} v_{j} \in E$, where $c, t$ are constants. By definition of degree of a vertex we have $d_{G}\left(v_{i}\right)=\left(d_{\widetilde{\mu_{P}}}\left(v_{i}\right), d_{\lambda_{Q}}\left(v_{i}\right)\right)=\left(\sum_{v_{i} v_{j} \in E} \widetilde{\mu_{Q}}\left(v_{i} v_{j}\right), \sum_{v_{i} v_{j} \in E} \lambda_{Q}\left(v_{i} v_{j}\right)\right)=\left(\sum_{v_{i} v_{j} \in E} c, \sum_{v_{i} v_{j} \in E} t\right)$ for all $v_{i} \in V$. Hence, $d_{G}\left(v_{i}\right)=(k c, k t)$. Therefore, $G$ is regular cubic graph. Now, $t d_{G}\left(v_{i} v_{j}\right)=\left(t d_{\widetilde{\mu_{Q}}}\left(v_{i} v_{j}\right), t d_{\lambda_{Q}}\left(v_{i} v_{j}\right)\right)$, where $t d_{t}\left(v_{i} v_{j}\right)=$ $\sum \widetilde{\mu_{Q}}\left(v_{i} v_{k}\right)+\sum \widetilde{\mu_{P}}\left(v_{k} v_{j}\right)+\widetilde{\mu_{Q}}\left(v_{i} v_{j}\right)$

$=\sum_{\substack{v_{i} v_{k} \in E \\ k \neq j}} c+\sum_{\substack{v_{k} v_{j} \in E \\ k \neq i}} c+c=c(k-1)+c(k-1)+c=c(2 k-1)$. Similarly, $t d_{\lambda_{Q}}\left(v_{i} v_{j}\right)=t(2 k-1)$, for all $v_{i} v_{j} \in E$. Hence, $G$ is also totally edge regular cubic graph.

Conversely, assume that $G$ is both regular and edge regular cubic graph. We prove that $\left(\widetilde{\mu_{Q}}, \lambda_{Q}\right)$ is a constant function. Since $G$ is regular, $d_{G}\left(v_{i}\right)=\left(c_{1}, c_{2}\right)$, for all $v_{i} \in V$. Also, $G$ is totally edge regular. Hence $t d_{G}\left(v_{i} v_{j}\right)=\left(t_{1}, t_{2}\right)$, for all $v_{i} v_{j} \in E$. By definition of totally edge degree we have $t d_{G}\left(v_{i} v_{j}\right)=\left(t d_{\widetilde{\mu_{Q}}}\left(v_{i} v_{j}\right), t d_{\lambda_{Q}}\left(v_{i} v_{j}\right)\right)$, where $t d_{G}\left(v_{i} v_{j}\right)=$ $d_{\widetilde{\mu_{P}}}\left(v_{i}\right)+d_{\widetilde{\mu_{P}}}\left(v_{j}\right)-\widetilde{\mu_{Q}}\left(v_{i} v_{j}\right)$, for all $v_{i} v_{j} \in E, t_{1}=c_{1}+c_{2}-\widetilde{\mu_{Q}}\left(v_{i} v_{j}\right)$. So, $\widetilde{\mu_{Q}}\left(v_{i} v_{j}\right)=2 c_{1}-t_{1}$. Similarly we have $\lambda_{Q}\left(v_{i} v_{j}\right)=2 c_{2}-t_{2}$, for all $v_{i} v_{j} \in E$. Hence, $\left(\widetilde{\mu_{P}}, \lambda_{Q}\right)$ is a constant function.

Definition 3.3. A cubic graph $G=\left(V=\left\{v_{1}, v_{2}, \cdots, v_{n}\right\}, E\right)$, is said to be strongly regular, if it satisfies the following axioms:

(i) $G$ is $k=\left(k_{1}, k_{2}\right)$-regular cubic graph

(ii) The sum of membership values and non-membership values of the common neighborhood vertices of any pair of adjacent vertices and non-adjacent vertices $v_{i}, v_{j}$ of $G$ has the same weight and is denoted by $\lambda=\left(\widetilde{\lambda_{1}}, \lambda_{2}\right), \delta=\left(\widetilde{\delta_{1}}, \delta_{2}\right)$, respectively.

Note 3.1. Any strongly cubic graph $G$ is denoted by $G=(n, k, \lambda, \delta)$.

Example 3.3. Consider the cubic graph $G=(V, E)$ in Figure 3, where $V=\{a, b, c\}$ and 安 $h \overline{\bar{e}} n,\left\{a h, \underline{h} q, q a \underline{k}\left(\widetilde{k_{1}}, k_{2}\right)=([0.2,0.4], 1.2)\right.$,

$\lambda=\left(\widetilde{\lambda_{1}}, \lambda_{2}, \lambda^{*}\right)=[(0.4,0.6), 1.2)$,

$\delta=\left(\widetilde{\delta}_{1}, \delta_{2}\right)=[(0,0), 0]$. Hence, $G$ is a strongly regular cubic graph.

Theorem 3.6. If $G=(V, E)$ is a complete cubic graph with $\left(t_{A}, f_{A}\right)$ and $\left(t_{B}, f_{B}\right)$ as constant functions, then $G$ is a strongly regular cubic graph. 
Proof. Let $G=(V, E)$ be a complete cubic graph where $V=\left\{v_{1}, v_{2}, \cdots, v_{n}\right\}$. Since $\widetilde{\mu_{P}\left(v_{i}\right)}, \lambda_{Q}\left(v_{i}\right), \widetilde{\mu_{P}\left(v_{i} v_{j}\right)}$ and $\lambda_{Q}\left(v_{i} v_{j}\right)$ are constant functions, hence, $\left.\widetilde{\mu_{P}}\left(v_{i}\right)=r, \lambda_{(} v_{i}\right)=s$, for all $v_{i} \in V$ and $\widetilde{\mu_{P}}\left(v_{i} v_{j}\right)=c$ and $\lambda_{Q}\left(v_{i} v_{j}\right)=t$, for all $v_{i} v_{j} \in E$ where $r, s, c, t$ are constants. To prove that $G$ is a strongly regular cubic graph, we have to show that $G$ is $k=\left(k_{1}, k_{2}\right)$-regular cubic graph and the adjacent vertices have the same common neighborhood $\lambda=\left(\lambda_{1}, \lambda_{2}\right)$ and non-adjacent vertices have the same common neighborhood $\delta=\left(\delta_{1}, \delta_{2}\right)$. Now, Since $G$ is complete; $d_{G}\left(v_{i}\right)=\left(d_{\widetilde{\mu_{P}}}\left(v_{i}\right), d_{\lambda_{Q}}\left(v_{i}\right)\right)=\left(\sum_{v_{i} v_{j} \in E} \widetilde{\mu_{P}}\left(v_{i} v_{j}\right)\right.$, $\left.\sum_{v_{i} v_{j} \in E} \lambda_{Q}\left(v_{i} v_{j}\right)\right)=((n-1) c,(n-1) t)$ Hence, $G$ is an $((n-1) c,(n-1) t)-$ regular cubic graph. Now, since $G$ is complete cubic graph, the sum of membership values and non-membership values of common neighborhood vertices of any pair of adjacent vertices $\lambda=((n-2) r,(n-2) s)$ are the same and the sum of membership values and non-membership values of common neighborhood vertices of any pair of non-adjacent vertices $\delta=0$ are the same.

Remark 3.2. If $G$ is a strongly regular disconnected cubic graph then, $\delta=0$.

Definition 3.4. A cubic graph $G=(V, E)$ is said to be a biregular cubic graph if it satisfies the following axioms:

(i) $G$ is $k=\left(k_{1}, k_{2}\right)$-regular cubic graph.

(ii) $V=V_{1} \cup V_{2}$ be the bipartition of $V$ and every vertex in $V_{1}$ has the same neighborhood degree $M=\left(M_{1}, M_{1}\right)$ and every vertex in $V_{2}$ has the same neighborhood degree $N=$ $\left(N_{1}, N_{2}\right)$, where $M$ and $N$ are constants.

Example 3.4. Consider a cubic graph $G=(V, E)$ in Figure 4 , where $V=\left\{u_{1}([0.25,0.35], 0.5)\right.$, $u_{2}([0.35,0.45], 0.6)$, $u_{3}([0.25,0.35], 0.5), u_{4}([0.35,0.45], 0.6)$, $u_{5}([0.35,0.45], 0.6), u_{6}([0.25,0.35], 0.5)$, $\left.u_{7}([0.35,0.45], 0.6), u_{8}([0.25,0.35], 0.5)\right\}$

and $E=\left\{u_{1} u_{2}, u_{1} u_{4}, u_{1} u_{5}, u_{2} u_{6}, u_{2} u_{3}, u_{3} u_{4}, u_{3} u_{7}, u_{4} u_{8}, u_{5} u_{6}, u_{5} u_{8}, u_{6} u_{7}, u_{7} u_{8}\right\}$. The membership values of the edges $\left(u_{1}, u_{2}\right),\left(u_{3}, u_{4}\right),\left(u_{5}, u_{8}\right),\left(u_{6}, u_{7}\right)$ is $([0.05,0.15], 0.6)$ and that of $\left(u_{1}, u_{4}\right),\left(u_{2}, u_{3}\right),\left(u_{5}, u_{6}\right),\left(u_{7}, u_{8}\right)$ is $([0.15,0.25], 0.7)$ and that of $\left(u_{1}, u_{5}\right),\left(u_{2}, u_{6}\right)$, $\left(u_{4}, u_{8}\right),\left(u_{3}, u_{7}\right)$ is

$\left([0.25,9.35],\left(k_{1}\right) 6 k_{2}\right) \stackrel{\text { Then }}{=}([0.55,0.65], 1.9), V_{1}=\left\{u_{1}, u_{3}, u_{6}, u_{8}\right\}, V_{2}=\left\{u_{2}, u_{4}, u_{5}, u_{7}\right\}, M=$ $\left(M_{1}, M_{2}\right)=([1.15,1.25], 1.8)$ and $N=\left(N_{1}, N_{2}\right)=([0.85,0.95], 1.5)$.

Theorem 3.7. If $G=(V, E)$ is a strongly regular cubic graph which is strong then, $\bar{G}$ is a $\left(k_{1}, k_{2}\right)$-regular.

Proof. Let $G=(V, E)$ be a strongly regular cubic graph. Then by definition, $G$ is a $\left(k_{1}, k_{2}\right)$-regular. Since $G$ is strong, we have

$$
\begin{aligned}
& \overline{\widetilde{\mu_{Q}}}\left(v_{i} v_{j}\right)= \begin{cases}0 & \text { for all } v_{i} v_{j} \in E \\
\min \left(\widetilde{\mu_{P}}\left(v_{i}\right), \widetilde{\mu_{P}}\left(v_{j}\right)\right) & \text { for all } v_{i} v_{j} \notin E\end{cases} \\
& \overline{\lambda_{Q}}\left(v_{i} v_{j}\right)= \begin{cases}0 & \text { for all } v_{i} v_{j} \in E \\
\max \left(\lambda_{P}\left(v_{i}\right), \lambda_{P}\left(v_{j}\right)\right) & \text { for all } v_{i} v_{j} \notin E .\end{cases}
\end{aligned}
$$




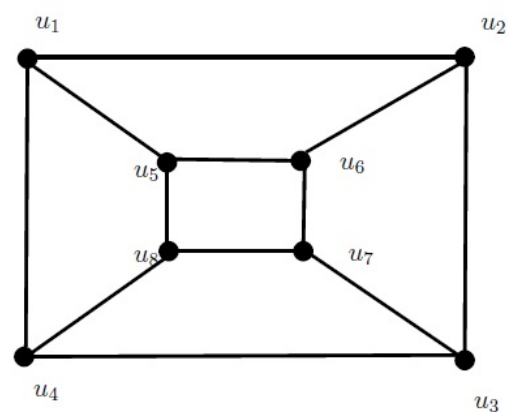

FIGURE 4. Biregular cubic graph

Now, since $G$ is strong, the degree of a vertex $v_{i}$ in $\bar{G}$ is $d_{\bar{G}}\left(v_{i}\right)=\left(d_{\overline{\mu_{p}}}\left(v_{i}\right), d_{\overline{\lambda_{p}}}\left(v_{i}\right)\right)$, where $d_{\overline{\mu_{P}}}\left(v_{i}\right)=\sum_{v_{i} \neq v_{j}} \overline{\mu_{Q}}\left(v_{i} v_{j}\right)=\sum_{v_{i} \neq v_{j}} \tilde{\mu}_{\bar{P}}\left(v_{i}\right) \wedge \widetilde{\mu}_{\bar{P}}\left(v_{j}\right)=k_{1}, \quad \forall v_{i} \in V$ Hence, $d_{\bar{G}}\left(v_{i}\right)=\left(k_{1}, k_{2}\right)$, for all $v_{i} \in V$. So, $\bar{G}$ is a $\left(k_{1}, k_{2}\right)$-regular cubic graph.

Theorem 3.8. Let $G=(V, E)$ be a strong cubic graph. Then $G$ is a strongly regular if and only if $\bar{G}$ is a strongly regular.

Proof. Assume that $G=(V, E)$ is a strongly regular cubic graph. Then $G$ is $\left(k_{1}, k_{2}\right)-$ regular and the adjacent vertices and the non-adjacent vertices have the same common neighborhood $\lambda=\left(\lambda_{1}, \lambda_{2}\right)$ and $\delta=\left(\delta_{1}, \delta_{2}\right)$, respectively. We have to prove that $\bar{G}$ is a strongly regular cubic graph. If $G$ is strongly regular cubic graph which is strong then $\bar{G}$ is a $\left(k_{1}, k_{2}\right)$-regular cubic graph by Theorem 3.7. Next, let $S_{1}$ and $S_{2}$ be the sets of all adjacent vertices and non-adjacent vertices of $G$. That is, $S_{1}=\left\{v_{i} v_{j} \mid v_{i} v_{j} \in E\right\}$, where $v_{i}$ and $v_{j}$ have same common neighborhood $\lambda=\left(\lambda_{1}, \lambda_{2}\right)$ and $S_{2}=\left\{v_{i} v_{j} \mid v_{i} v_{j} \notin E\right\}$, where $v_{i}$ and $v_{j}$ have same common neighborhood $\delta=\left(\delta_{1}, \delta_{2}\right)$. Then, $\overline{S_{1}}=\left\{v_{i} v_{j} \mid v_{i} v_{j} \in \bar{E}\right\}$, where $v_{i}$ and $v_{j}$ have same common neighborhood $\delta=\left(\delta_{1}, \delta_{2}\right)$ and $\overline{S_{2}}=\left\{v_{i} v_{j} \mid v_{i} v_{j} \notin \bar{E}\right\}$, where $v_{i}$ and $v_{j}$ have same common neighborhood $\lambda=\left(\lambda_{1}, \lambda_{2}\right)$. Which implies $\bar{G}$ is a strongly regular. Similarly we can prove the converse.

Theorem 3.9. A strongly regular cubic graph $G$ is a biregular cubic graph if the adjacent vertices have the same common neighborhood $\lambda=\left(\lambda_{1}, \lambda_{2}\right) \neq 0$ and the non-adjacent vertices have the same common neighborhood $\delta=\left(\delta_{1}, \delta_{2}\right) \neq 0$.

Proof. Let $G=(V, E)$ be a strongly regular cubic graph. Then we have $d\left(v_{i}\right)=$ $\left(k_{1}, k_{2}\right)$, for all $v_{i} \in V$. Assume that the adjacent vertices have the same common neighborhood $\delta=\left(\delta_{1}, \delta_{2}\right) \neq 0$. Let $S$ be the sets of all non-adjacent vertices. That is $S=\left\{v_{i} v_{j} \mid v_{i}\right.$ is not adjacent to $\left.v_{j}, i \neq j, v_{i}, v_{j} \in V\right\}$. Now the vertex partition of $G$ is $V_{1}=\left\{v_{i} \mid v_{i} \in S\right\}$ and $V_{2}=\left\{v_{j} \mid v_{j} \in S\right\}$. Then $V_{1}$ and $V_{2}$ have the same neighborhood degree, since $G$ is a strongly regular. Hence, $G$ is a bi-regular cubic graph.

Definition 3.5. (i) If the underlying graph $G^{*}$ is an edge regular graph, then $G$ is said to be a partially edge regular cubic graph. 
(ii) If $G$ is both edge regular and partially edge regular cubic graph, then $G$ is said to be a full edge regular cubic graph.

Theorem 3.10. Let $G$ be a cubic graph on $G^{*}$ such that $\left(t_{B}, f_{B}\right)$ is a constant function. If $G$ is full regular, then $G$ is full edge regular cubic graph.

Proof. Let $\left(\widetilde{\mu_{Q}}, \lambda_{Q}\right)$ be a constant function. Then, $\widetilde{\mu_{Q}}\left(v_{i} v_{j}\right)=c_{1}$ and $\lambda_{Q}\left(v_{i} v_{j}\right)=c_{2}$, for every $v_{i} v_{j} \in E$ where $c_{1}$ and $c_{2}$ are constants. Suppose that $G$ is full regular cubic graph then $d_{G}\left(v_{i}\right)=\left(k_{1}, k_{2}\right)=k$ and $d_{G^{*}}\left(v_{i}\right)=r$, for all $v_{i} \in V$, where $k$ and $r$ are constants. $d_{G^{*}}\left(v_{i} v_{j}\right)=d_{G^{*}}\left(v_{i}\right)+d_{G^{*}}\left(v_{j}\right)-2=2 r-2=$ constant. Hence, $G^{*}$ is an edge regular graph. Now, since $G$ is regular, $d_{G}\left(v_{i} v_{j}\right)=\left(d_{\widetilde{\mu_{Q}}}\left(v_{i} v_{j}\right), d_{\lambda_{Q}}\left(v_{i} v_{j}\right)\right)$, for all $v_{i} v_{j} \in E$ where $d_{\widetilde{\mu_{Q}}}\left(v_{i} v_{j}\right)$ $=d_{\widetilde{\mu_{P}}}\left(v_{i}\right)+d_{\widetilde{\mu_{P}}}\left(v_{j}\right)-2 \widetilde{\mu_{Q}}\left(v_{i} v_{j}\right)=k_{1}+k_{1}-2 c_{1}=2 k_{1}-2 c_{1}=$ constant . Similarly, for all $v_{i} v_{j} \in E, d_{\lambda_{Q}}\left(v_{i} v_{j}\right)=2 k_{2}-2 c_{2}=$ constant. Hence, $G$ is an edge regular cubic graph. Therefore, $G$ is full edge regular cubic graph.

Theorem 3.11. Let $G$ be a t-totally edge regular cubic graph and $t_{1}$-partially edge regular cubic graph. Then $S(G)=\frac{q t}{1+t_{1}}$, where $q=|E|$.

Proof. The size of cubic graph $G$ is

$$
S(G)=\left(\sum_{v_{i} v_{j} \in E} \widetilde{\mu_{Q}}\left(v_{i} v_{j}\right), \sum_{v_{i} v_{j} \in E} \lambda_{Q}\left(v_{i} v_{j}\right)\right) .
$$

Since $G$ is $t$-totally edge regular cubic graph i.e., $t d_{G}\left(v_{i} v_{j}\right)=t$ and $G^{*}$ is $t_{1}$-partially edge regular cubic graph i.e. $d_{G^{*}}\left(v_{i} v_{j}\right)=t_{1}$. Thus, $\sum t d_{G}\left(v_{i} v_{j}\right)=\left(\sum_{v_{i} v_{j} \in E} d_{G^{*}}\left(v_{i} v_{j}\right) \widetilde{\mu_{Q}}\left(v_{i} v_{j}\right)\right.$, $\left.\sum_{v_{i} v_{j} \in E} d_{G^{*}}\left(v_{i} v_{j}\right) \lambda_{Q}\left(v_{i} v_{j}\right)\right)+S(G) . q t=t_{1} S(G)+S(G)$. Hence, $S(G)=\frac{q t}{1+t_{1}}$.

\section{CUBIC DIGRAPH IN TRAVEL TIME}

In modern age, planning efficient routes is essential for industry and business, with applications as varied as product distribution, laying new fiber optic lines for broadband internet, and suggesting new friends within social network websites such as Facebook. When we visit a website like Google Maps and looking for directions from one city to another city in USA, we are usually asking for a shortest path between the two cities. These computer applications use representations of the road maps as graphs, with estimated travel times as edge weights. The travel time is a function of traffic density on the road or the length of the road. The traffic density is a fuzzy, while the length of a road is a crisp quantity. In a road network, crossings are represented by vertices, roads by edges and traffic density on the road is usually calculated between adjacent crossings. These factors can be represented as a cubic set. Any model of a road network can be represented as a cubic digraph $D=(C, R)$, where $C$ is a cubic set of crossings(vertices) at which the traffic density is calculated and connectivity conditions as truth-membership degree with intervals $\widetilde{\mu_{P}}(x)$ and falsity membership degree $\lambda_{P}(x)$

$C=\{(a,[0.2,0.3], 0.4),(b,[0.3,0.5], 0.6),(c,[0.5,0.7], 0.8)$,

$(d,[0.4,0.6], 0.7),(e,[0.2,0.5], 0.6),(f,[0.3,0.4], 0.5),(g,[0.4,0.6], 0.7)\}$

and $\mathrm{R}$ is a cubic set of roads (edges) between crossings whose truth membership degree 
12 K. Kumar.P.K, H. Rashmanlou, A.A. Talebi, and F. Mofidnakhaei

$\widetilde{\mu_{P}}(x)$ and false membership degree $\lambda_{P}(x)$ can be calculated as:

$\widetilde{\mu_{Q}}(x y) \leq \min \left\{\widetilde{\mu_{P}}(x), \widetilde{\mu_{P}}(y)\right\}$

$\lambda_{P}(x y) \geq \max \left\{\lambda_{P}(x), \lambda_{P}(y)\right\}$ for all $x y \in E$.

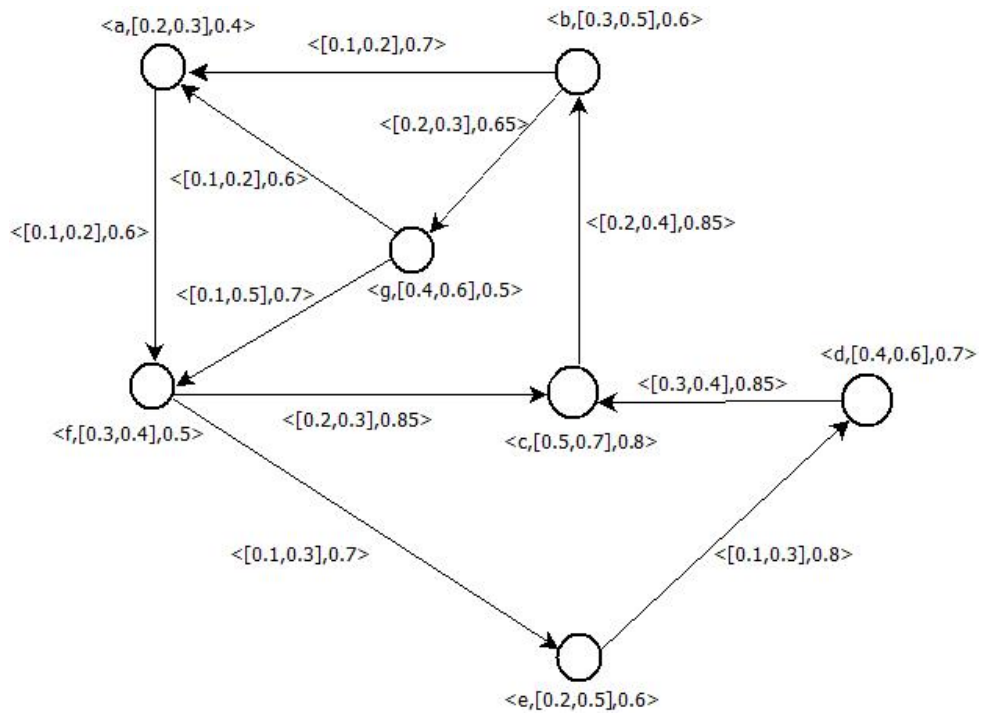

FIGURE 5. Cubic digraph of a road network

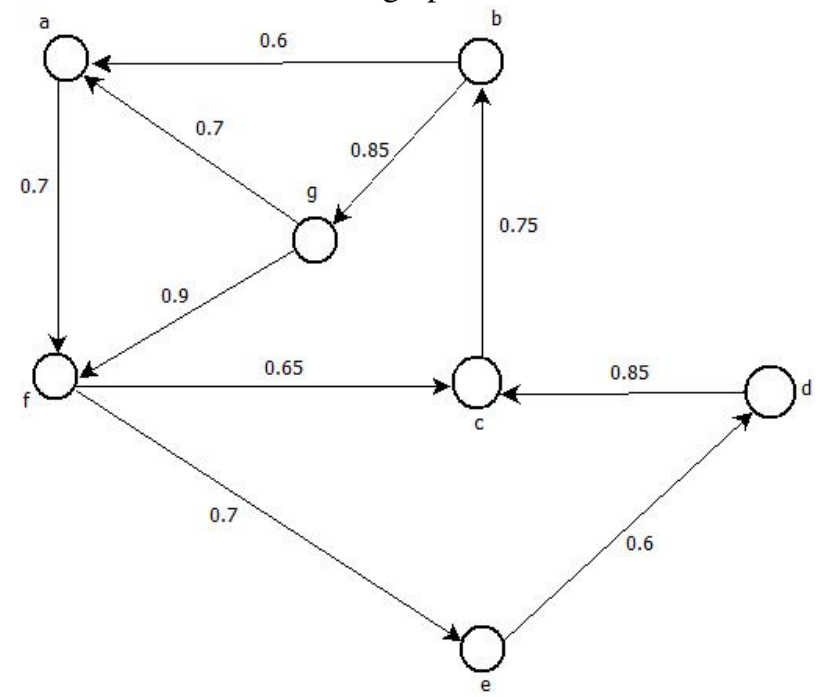

FIGURE 6. Weighted digraph of a road network 
The cubic digraph $D=(C, R)$ of the travel time is given in Figure 5. The cubic out neighborhood are given in Table 4 .

The final weights on edges can be calculated by finding the score function of cubic edges as

$s_{i}=\left(\widetilde{\mu_{R}}\right)_{i}+1-\left(\lambda_{R}\right)_{i}$. The final weighted digraph given in Figure 6 which can be used for finding the shortest or optimal path between two locations (vertices) by any of the known methods, like Floyd's algorithm, Djikstra's and A star algorithm. Weighted relations are given in Table 4.

\begin{tabular}{|l|l|}
\hline Crossings & $N^{+}$(crossings) \\
\hline a & $\{\mathrm{f},([0.1,0.2], 0.6)\}$ \\
$\mathrm{b}$ & $\{\mathrm{a},([0.1,0.2], 0.7), \mathrm{g},([0.2,0.3], 0.65)\}$ \\
$\mathrm{c}$ & $\{\mathrm{b},([0.2,0.4], 0.85)\}$ \\
d & $\{\mathrm{c},([0.3,0.4], 0.85)\}$ \\
$\mathrm{e}$ & $\{\mathrm{d},([0.1,0.3], 0.8)\}$ \\
$\mathrm{f}$ & $\{\mathrm{c},([0.2,0.3], 0.85), \mathrm{e},([0.1,0.3], 0.7)\}$ \\
$\mathrm{g}$ & $\{\mathrm{a},([0.1,0.2], 0.6), \mathrm{f},([0.1,0.5], 0.7)\}$ \\
\hline
\end{tabular}

\begin{tabular}{|l|l|}
\hline Crossings & $N^{+}$(crossings) \\
\hline a & $\{\mathrm{f}, 0.7\}$ \\
b & $\{\mathrm{a}, 0.6, \mathrm{~g}, 0.85\}$ \\
$\mathrm{c}$ & $\{\mathrm{b}, 0.75\}$ \\
$\mathrm{d}$ & $\{\mathrm{c}, 0.85\}$ \\
$\mathrm{e}$ & $\{\mathrm{d}, 0.6\}$ \\
$\mathrm{f}$ & $\{\mathrm{c}, 0.65, \mathrm{e}, 0.7\}$ \\
$\mathrm{g}$ & $\{\mathrm{a}, 0.7, \mathrm{f}, 0.9\}$ \\
\hline
\end{tabular}

The following algorithm generates the weighted digraph, WR, for given cubic digraph and resolves to obtain the optimal path from a source vertex to destination vertex.

\section{CONCLUSION}

Fuzzy graph theory has numerous applications in modern science and technology, especially in the fields of operations research, neural networks, and decision making. Since the cubic models give more precision, flexibility and compatibility to the system as compared to the classical and fuzzy models, in this paper the definition of partial edge regular and fully edge regular cubic graph are given and some properties of edge regular cubic graph are studied. Also, we have introduced the condition under which edge regular cubic graph and totally edge regular cubic graph are equivalent. In our future work, we are going to extend the properties of strongly edge regular cubic graph in matrix representation. 


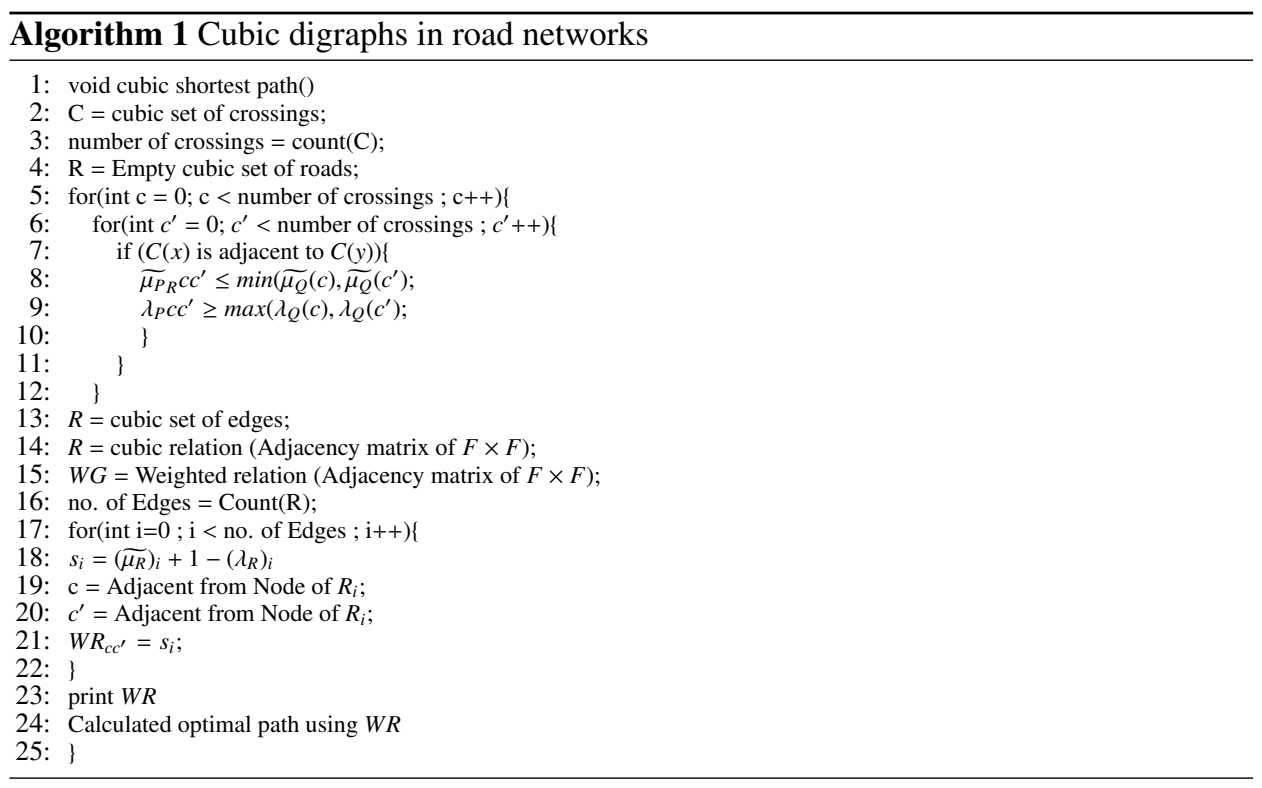

\section{REFERENCES}

[1] M. Akram and W. A. Dudec, "Interval-valued fuzzy graphs", Computers and Mathematics with Applications, 61 (2011), 289-299.

[2] P. Bhattacharya, "Some remarks on fuzzy graphs", Pattern Recognition Letters, 6 (1987), 297-302.

[3] J. Hongmei, W. Lianhua, "Interval-valued fuzzy subsemigroups and subgroups associated by interval-valued fuzzy graphs", in: WRI Global Congress on Intelligent Systems, (2009),484-487.

[4] Y. B. Jun, Ch. S. Kim, K. O. Yang, "Cubic sets", Annals of Fuzzy Mathematics and Informatics, 4 (1) (2012), 83-98.

[5] A. Kauffman, "Introduction a la theorie des sous-emsembles 503 flous", Masson et Cie 1 (1973).

[6] M. G. Karunambigai, K. Palanivel and S. Sivasankar, "Edge regular intuitionistic fuzzy graph", Advances in Fuzzy Sets and Systems, 20 (1) (2015), 25-46.

[7] L. J. Kohout, W. Bandler, "Fuzzy interval inference utilizing the checklist paradigm and BK relational products", in: R.B. Kearfort et al. (Eds.), Applications of Interval Computations, Kluwer, Dordrecht, (1996), 291-335.

[8] J. N. Mordeson, C. S. Peng, “Operations on fuzzy graphs”, Information Sciences, 79 (1994), 159-170.

[9] J. N. Mordeson, P. S. Nair, "Fuzzy Graphs and Fuzzy Hypergraphs", Physica Verlag, Heidelberg, (1998).

[10] H. Rashmanlou and M. Pal, "Some properties of highly irregular interval-valued fuzzy graphs", World Applied Sciences Journal, 27 (12) (2013), 1756-1773.

[11] H. Rashmanlou, S. Samanta, M. Pal and R. A. Borzooei, “A study on bipolar fuzzy graphs”,Journal of Intelligent and Fuzzy Systems, 28 (2015), 571-580.

[12] H. Rashmanlou, S. Samanta, M. Pal and R. A. Borzooei, "Bipolar fuzzy graphs with categorical properties", International Journal of Computational Intelligent Systems, 8 (5) (2015), 808-818.

[13] A. Rosenfeld, Fuzzy graphs, in: L.A. Zadeh, K.S. Fu, M. Shimura (Eds.), "Fuzzy Sets and Their Applications", Academic Press, New York, (1975), 77-95.

[14] R. Sambuc, "Functions -Flous", Application alaide au Diagnostic en Pathologie Thyroidienne, These de Doctorat en Medecine, Marseille, (1975).

[15] M. S. Sunitha, A. Vijayakumar, "Complement of a fuzzy graph", Indian Journal of Pure and Applied Mathematics, 33 (2002), 1451-1464. 
[16] S. Samanta, M. Pal and M. Akram,“ $m$-step fuzzy competition graphs”, Journal of Applied Mathematics and Computing, 47 (2015), 461472.

[17] S. Samanta and M. Pal, "Fuzzy tolerance graphs", International Journal Latest Trend Mathematics, 1 (2) (2011), 57-67.

[18] S. Samanta and M. Pal, "Fuzzy threshold graphs", CiiT International Journal of Fuzzy Systems, 3 (12) (2011), 360-364.

[19] S. Samanta and M. Pal, "Fuzzy k-competition graphs and p-competition fuzzy graphs", Fuzzy Engineering and Information, 5 (2) (2013), 191-204.

[20] S. Samanta, M. Pal and A. Pal, "New concepts of fuzzy planar graph", International Journal of Advanced Research in Artificial Intelligence, 3 (1) (2014), 52-59.

[21] I. B. Turksen, "Interval-valued strict preference with Zadeh triples", Fuzzy Sets and Systems, 78 (1996), 183-195.

[22] L. A. Zadeh, "Fuzzy sets, Information and Control”, 8 (1965), 338-353.

[23] L. A. Zadeh, "The concept of a linguistic variable and its application to approximate reasoning-I", Information Sciences, 8 (1975), 199-249. 